\title{
Modeling flame extinction and reignition in large eddy simulations with fast chemistry
}

\author{
J.P. White ${ }^{\mathrm{a}, *}$, S. Vilfayeau ${ }^{\mathrm{a}}$, A.W. Marshall ${ }^{\mathrm{a}}$, A. Trouvéa ${ }^{\mathrm{a}}$, R.J. McDermott ${ }^{\mathrm{b}}$ \\ ${ }^{a}$ Department of Fire Protection Engineering, University of Maryland, \\ College Park, MD 20742, USA \\ ${ }^{b}$ National Institute of Standards and Technology, Gaithersburg, MD 20899, USA
}

\begin{abstract}
This work seeks to support the validation of large eddy simulation models used to simulate fire suppression. The emphasis in the present study is on the prediction of flame extinction and the prevention of spurious reignition using a fast chemistry, mixing-controlled combustion model applicable to realistic fire scenarios of engineering interest. The configuration provides a buoyant, turbulent methane diffusion flame within a controlled co-flowing oxidizer. The oxidizer allows for the supply of a mixture of air and nitrogen, including conditions for which oxygen-dilution in the oxidizer leads to flame extinction. Measurements to support model validation include local profiles of thermocouple temperature and oxygen mole fraction, global combustion efficiency, and the limiting oxygen index. The present study evaluates the performance of critical flame temperature based extinction and reignition models using the Fire Dynamics Simulator, an open-source fire dynamics solver. Alternate model cases are explored, each offering a unique treatment of extinction and reignition. Comparisons between simulated results and experimental measurements are used to evaluate the capability of these models to accurately describe flame extinction. Of the considered cases, those that include provisions to prevent spurious reignition show excellent agreement with measured data, whereas a baseline case lacking explicit reignition treatment fails to predict extinction.
\end{abstract}

\footnotetext{
* Corresponding author

Email address: jwhite21@umd.edu (J.P. White)
}

Preprint submitted to Fire Safety Journal

March 19, 2017

(C) 2016. This manuscript version is made available under the Elsevier user license http://www.elsevier.com/open-access/userlicense/1.0/ 
Keywords: Extinction, Fast Chemistry, Fire Dynamics Simulator, Large

Eddy Simulation, Mixing Controlled Combustion, Reignition

1

\section{Introduction}

The numerical simulation of fire phenomena using classical computational fluid dynamics (CFD) methods has advanced significantly over recent years. This progress has led to an increasing demand for the use of CFD tools to analyze and predict the performance of fire protection systems. For fire suppression applications in particular, this demand remains largely unmet as modern CFD solvers have not yet been shown to adequately model fire suppression physics in configurations of practical interest, namely in the buoyant turbulent diffusion flames characteristic of most fire safety applications. This limitation is the result of the complex physical processes that govern turbulent fire suppression, comprising both localized extinction events and the potential reignition of unburned fuel following such extinction events. Neither of these phenomena are easily modeled. Further contributing to the issue is the general unavailability of detailed experimental data suitable for model validation, which also inhibits model development.

In recognition of these constraints, numerous experimental and computational studies have investigated the extinction behaviors of diffusion flames in various configurations. These works have successfully identified the primary physical mechanisms for extinction (thermal, aerodynamic, and kinetic quenching) [1-9], while others have made progress toward developing simple formulations to model flame extinction in cases applicable to realistic fire scenarios [10-15]. Additional studies have highlighted the primary features of flame reignition events, which may follow localized extinction in large-scale turbulent flames [16-21].

As noted in previous works, the primary difficulty associated with modeling flame extinction and reignition in fire applications is that both phenomena are controlled by small-scale quantities, including the flame temperature and the 
fuel-oxidizer mixing rate at the flame sheet, that cannot be resolved numerically in configurations of practical interest. In particular, the present study focuses on models that are specifically applicable to fire safety applications, i.e. models that are applicable to complex large-scale configurations where detailed fuel chemistry is typically unknown. Such models primarily comprise large eddy simulation (LES) approaches incorporating a combustion model based on the classical eddy-dissipation concept (EDC), wherein reactions are controlled by turbulent mixing and without regard for chemical kinetics. These modeling choices represent the most appropriate selections for simulating fire phenomena in practical engineering applications because alternative direct numerical simulations (DNS) cannot be applied to large-scale configurations of engineering interest, and the combustion of realistic fuel sources cannot be represented using detailed finite-rate chemical kinetics models. Available treatments for modeling flame extinction phenomena in these scenarios include models based on a critical flame temperature $[10,11]$ or models based on a critical flame Damköhler number [6, 7, 12-15].

Critical flame temperature based models are often simpler and computationally less expensive, though such models do not consider the effect of chemical time scales. Such models are therefore incapable of modeling aerodynamic quenching effects and are unsuitable for configurations with high-strain flames [12]. By comparison, Damköhler number based extinction models incorporate additional physics to account for chemical time scales and aerodynamic quenching effects. For this reason, Damköhler number based extinction models may be expected to be more accurate $[7,12,13]$; however, critical flame temperature based models may also be expected to perform adequately in low-strain flame configurations where chemical time scale effects may be safely ignored [11].

While flame extinction modeling has achieved notable advancement over recent years, numerical studies focused on flame reignition phenomena are relatively few. As a result, available models for the treatment of flame reignition are presently limited to critical temperature based models [14, 15]. This limitation is compounded by relatively unstudied issues concerning how the model 
should distinguish between piloted and non-piloted fuel sources, or between primary ignition at the fuel source and reignition occurring downstream of localized extinction events in the bulk flow. Such issues are particularly relevant to LES models incorporating EDC combustion because combustion is controlled by mixing and without regard for the initial temperature of the reactants. In such applications, careful consideration for reignition must be provided, otherwise spurious reignition may result, presently defined as the simulated reignition of previously extinguished combustible mixtures occurring at non-physical low temperatures.

The present work provides a detailed investigation of spurious reignition and its significance in affecting simulated extinction performance. The selected configuration includes recent experimental data for the suppression of a buoyant turbulent methane diffusion flame via nitrogen dilution of the oxidizer $[22,23]$. This simplified, but well-characterized configuration incorporates the essential features of a suppressed accidental fire (low-strain buoyancy-driven flow, turbulence, intense radiative emissions), while isolating the flame extinction physics of interest. In particular, simulations in the selected configuration highlight conditions for which localized flame extinction events may be followed by reignition events.

The present study utilizes the Fire Dynamics Simulator (FDS) [24], an opensource CFD solver that is widely used throughout the fire safety consulting and design industries. The selected numerical framework incorporates a LES approach utilizing the classical EDC treatment for mixing-controlled combustion. Selected models for flame extinction and reignition use the concept of a critical flame temperature, which should be viewed as an empirically-determined fuelspecific quantity approximating the flame temperature at the limits of flammability (considering heat losses) [25].

The present work follows a separate recent numerical study of the same configuration, but incorporating a robust Damköhler number based extinction model and a simple critical temperature based reignition model [15]. Results from that study suggest that simulated extinction performance in the present 
configuration is dominated by the reignition model and that the quality of the extinction model may therefore be of minor importance. The present work extends the study presented in [15] by evaluating whether a much-simplified critical flame temperature based extinction model can achieve similar performance in the selected low-strain configuration. The present investigation of spurious reignition then attempts to explain the dominance of the reignition model.

Results from the present work demonstrate the potential impairment that spurious reignition can cause to simulated extinction performance when its effects are not considered. To prevent such impairment, a pair of simple options for reignition modeling are developed and validated. The first is a novel concept featuring a spatially variable reignition criterion that permits fuel sources to be explicitly defined as either piloted or non-piloted. The second is an adaptation of the tiered reaction concept proposed in [15], which permits separation of ignition, extinction, and reignition via a split reaction mechanism. The present work additionally provides a validation study for the combination of these reignition treatments with the existing critical flame temperature based extinction model provided in the FDS solver.

\section{Modeling Approach}

\subsection{Solver}

The Fire Dynamics Simulator (FDS) provides a variable density, low Machnumber Navier-Stokes solver tuned for buoyancy-driven flows with heat release. FDS features a block-structured, Cartesian staggered grid and a parallel computing capability using MPI and OpenMP protocols. The numerics are generally second-order accurate with explicit time integration. Combustion is firstorder accurate and time-split from transport, consistent with the classical eddydissipation concept (EDC) model [26, 27]. As applied in the present work, combustion is treated with a single-step, global reaction involving lumped species for the fuel, oxidizer, and products [28]. 
A large eddy simulation (LES) framework is employed for subgrid-scale turbulence modeling, where the LES residual stress and transport terms are closed via gradient diffusion coupled with a modified version of the Deardorff eddyviscosity model [29], using an algebraic model for the subgrid kinetic energy based on scale similarity [30], and constant values for the turbulent Schmidt and Prandtl numbers.

FDS models thermal radiation via the radiative transfer equation using a discrete-ordinates, finite-volume method with gray absorption properties [31]. In the present study, the description of thermal radiation is further simplified by modeling flame emission using the concept of a global radiative loss fraction $\left(\chi_{\text {rad }}\right)$; where $\chi_{\text {rad }}$ is prescribed in the simulations a priori from experimental measurements.

For a comprehensive discussion detailing the mathematical models employed in the FDS solver, the reader is referred to the FDS technical reference guide [32-34]. Particularly relevant to the present work, the local volumetric heat release rate due to combustion, $\dot{Q}^{\prime \prime \prime}$, is given by

$$
\dot{Q}^{\prime \prime \prime}=-\sum_{k} \dot{m}_{k}^{\prime \prime \prime} \Delta h_{f, k}^{\circ}
$$

where $\dot{m}_{k}^{\prime \prime \prime}$ is the volumetric mass reaction rate and $\Delta h_{f, k}^{\circ}$ the mass-specific standard enthalpy of formation for each species, $k$, present in the prescribed combustion reaction. The volumetric mass reaction rate of fuel, $\dot{m}_{f u e l}^{\prime \prime \prime}$, is evaluated as

$$
\dot{m}_{f u e l}^{\prime \prime \prime}=-\bar{\rho} \frac{\min \left(\tilde{Y}_{f u e l}, \tilde{Y}_{\text {ox }} / s\right)}{\tau_{\text {mix }}},
$$

where $\bar{\rho}$ is the local mass density, $\tilde{Y}_{f u e l}$ and $\tilde{Y}_{o x}$ are the local mass fractions of fuel and oxidizer, and $s$ is the stoichiometric oxidizer-to-fuel mass ratio. Other $\dot{m}_{k}^{\prime \prime \prime}$ are then determined from $\dot{m}_{f u e l}^{\prime \prime \prime}$ based on prescribed stoichiometric reaction coefficients.

In Eq. 2, $\tau_{m i x}$ is a modeled characteristic mixing time-scale with alternate definitions based on the resolution of the flow-field [35]. For all numerical resolutions considered in the present study, $\tau_{m i x}$ is governed by turbulent advection 
and defined as

$$
\tau_{\text {mix }}=\frac{C_{u} \Delta}{\sqrt{(2 / 3) k_{s g s}}},
$$

where $C_{u}=0.4$ is a model coefficient calibrated to match experimental flame height measurements [34], $\Delta=(\Delta x \Delta y \Delta z)^{1 / 3}$ is the LES filter width defined by the local grid resolution $(\Delta x, \Delta y, \Delta z)$, and $k_{s g s}$ is the modeled subgrid turbulent kinetic energy derived from the turbulence sub-model.

Using the Deardorff model [29], the turbulent viscosity, $\mu_{t}$, is given by

$$
\mu_{t}=\bar{\rho} C_{v} \Delta \sqrt{k_{s g s}}
$$

where $C_{v}=0.1$ is a model coefficient set to match a value reported in the literature [36]. Turbulent transport coefficients for mass and energy are then derived from $\mu_{t}$ via constant Schmidt and Prandtl numbers, $S c_{t}=P r_{t}=0.5$.

The subgrid turbulent kinetic energy is evaluated as

$$
k_{s g s}=\frac{1}{2}\left([\tilde{u}-\hat{u}]^{2}+[\tilde{v}-\hat{v}]^{2}+[\tilde{w}-\hat{w}]^{2}\right),
$$

where $[\tilde{u}, \tilde{v}, \tilde{w}]$ are the cell-mean values of the $x, y$, and $z$-components of the local velocity field, and $[\hat{u}, \hat{v}, \hat{w}]$ are weighted averages of their respective cell-mean counterparts over the adjacent computational cells (representing a test-filtered velocity field over length scale $2 \Delta$ ) [30,32].

\subsection{Configuration}

The configuration for the present study follows the experimental facility developed by White et al., illustrated in Fig. 1 [22, 23]. This configuration provides the suppression of a buoyant, turbulent, methane diffusion flame via nitrogen dilution of the oxidizer. Flames are stabilized above a 5 -cm-wide by $50-\mathrm{cm}-\mathrm{cong}$ slot burner surrounded by a 50 -cm-wide by 75 -cm-long co-flowing oxidizer. Surrounding the fuel port is a thin 5-cm-wide strip of ceramic fiberboard, which encourages horizontally directed entrainment at the flame base and promotes transition to fully turbulent conditions within the flame. Methane flows at a fixed rate of $1.0 \mathrm{~g} / \mathrm{s}$ to yield a flame with an unsuppressed total heat release rate of $50 \mathrm{~kW}$. 


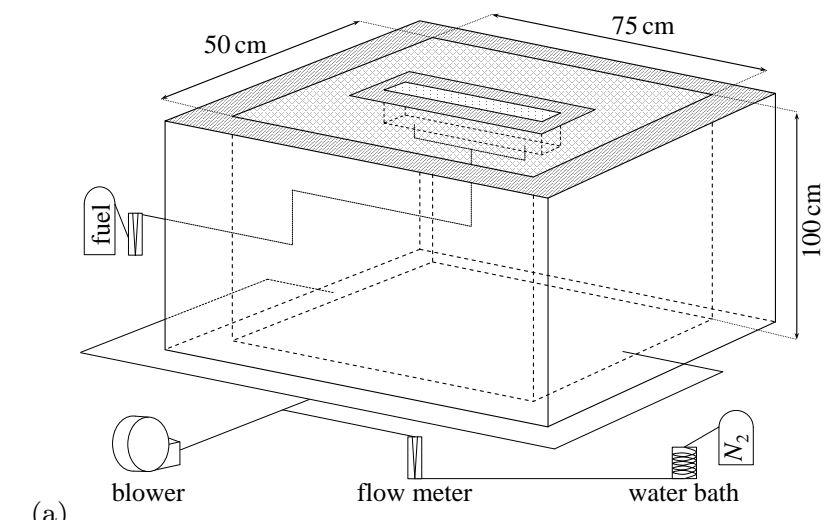

(a)

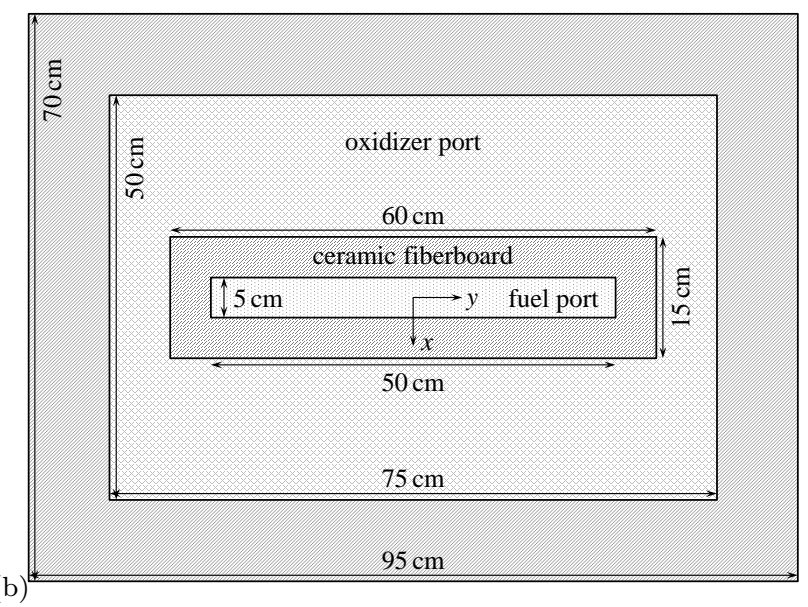

Figure 1: (a) Diagram of experimental facility. (b) Plan-view of fuel/oxidizer port surface.

The co-flowing oxidizer is supplied at a fixed rate of $85 \mathrm{~g} / \mathrm{s}$, including a variable flow of nitrogen gas mixed into the oxidizer to provide controlled flame suppression. Suppression potential is monitored via the oxygen mole-fraction in the oxidizer stream $\left(X_{O_{2}}^{o x}\right)$, measured using a sampling probe connected to a Servomex $540 \mathrm{E}$ paramagnetic oxygen analyzer (uncertainty $\pm 1250 \mathrm{ppm}$; response time $\pm 5 \mathrm{~s}$ ). The flow rate of nitrogen supplied to the oxidizer varies between $0-40 \mathrm{~g} / \mathrm{s}$ yielding changes in $X_{O_{2}}^{o x}$ from the ambient $X_{O_{2}}^{o x}=0.21$ to as low as $X_{O_{2}}^{o x}=0.11$, a value sufficient to achieve global flame extinguishment.

Available measurement data to support model validation include local ther- 


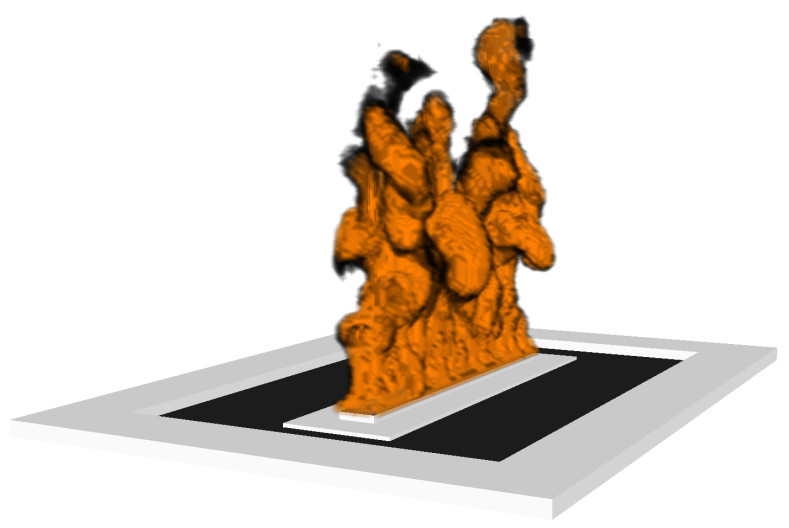

Figure 2: Illustration of the numerical configuration; a $50 \mathrm{~kW}$ methane diffusion flame is visualized where the local volumetric heat release rate exceeds $200 \mathrm{~kW} / \mathrm{m}^{3}$.

mocouple temperatures, measured via exposed-junction $1.0 \mathrm{~mm}$ bead-diameter K-type thermocouple probes (uncertainty $\pm 2 \mathrm{~K}$; response time $\pm 3 \mathrm{~s}$ ); local oxygen mole fractions, measured using a sampling probe connected to the aforementioned oxygen analyzer; global radiative loss fraction, measured via heat flux transducer combined with infrared flame imaging and an analytical multipoint radiation source model [22]; global combustion efficiency, measured via carbon dioxide generation calorimetry [23]; and the value of $X_{\mathrm{O}_{2}}^{o x}$ at extinction, termed the limiting oxygen index $(L O I)$.

The numerical configuration is depicted in Fig. 2, which visualizes the simulated flame at ambient condition $\left(X_{O_{2}}^{o x}=0.21\right)$. The computational domain is a 0.8 -m-wide by 1.0 -m-long by 1.0-m-tall box $(x \times y \times z)$, which is large enough to prevent excessive boundary-condition effects on the simulated flame. The baseline numerical grid features a uniform resolution in each coordinate direction with $\Delta x=\Delta y=\Delta z=5.0 \mathrm{~mm}$. The total number of computational cells is 6.4 million.

All edges of the computational domain feature an open, passive-flow boundary condition, with a background species of ambient air. The boundary conditions at the fuel and oxidizer ports utilize a prescribed mass-flux boundary condition (accounting for both convective and diffusive components), with values 
specified to match the experiment (fuel mass flow, $\dot{m}_{f u e l}=1.0 \mathrm{~g} / \mathrm{s}$, and oxidizer mass flow, $\left.\dot{m}_{o x}=85 \mathrm{~g} / \mathrm{s}\right)$. The fuel and oxidizer, as well as any ambient entrainment, enter the domain with a constant temperature of $T_{\infty}=293 \mathrm{~K}$. All solid surfaces within the domain, comprising the structural rim of the oxidizer port and the ceramic fiberboard around the fuel port, are described as inert, isothermal objects with constant temperature, $T_{\infty}=293 \mathrm{~K}$, and with zero-flow boundary condition. At initial condition, the interior of the domain includes a uniform composition of air at $T_{\infty}=293 \mathrm{~K}$ with ambient relative humidity of $40 \%$.

The value of $X_{\mathrm{O}_{2}}^{o x}$ at the oxidizer-port boundary is varied by controlling the relative mass fluxes of air and nitrogen leaving the oxidizer-port, which are balanced to maintain a constant total oxidizer flow rate, $\dot{m}_{o x}=85 \mathrm{~g} / \mathrm{s}$. At the start of each simulation $(t=0 \mathrm{~s}), X_{\mathrm{O}_{2}}^{o x}=0.21$, remaining constant at this value for the first $5 \mathrm{~s}$ of simulation time, a duration sufficient to achieve steady conditions in the flame and oxidizer flow fields.

After $t=5 \mathrm{~s}$, the value of $X_{\mathrm{O}_{2}}^{o x}$ is steadily decreased at a rate of $d X_{\mathrm{O}_{2}}^{o x} / d t=$ $0.002 \mathrm{~s}^{-1}$. Simulations are conducted for a total duration of $60 \mathrm{~s}$ so that $X_{O_{2}}^{o x}=$ 0.10 at the end of the simulation, a value sufficient to achieve global flame extinction. Simultaneous with the reduction in $X_{\mathrm{O}_{2}}^{o x}$, the value of $\chi_{\mathrm{rad}}$ also steadily decreases over the course of the simulation, with a prescribed trend matching experimental data [22].

In addition to transient simulations, which feature continuously variable $\mathrm{X}_{\mathrm{O}_{2}}^{o x}$, alternative steady-state simulations are conducted to provide converged turbulence statistics at discrete $X_{\mathrm{O}_{2}}^{o x}$ conditions. For such simulations, $X_{\mathrm{O}_{2}}^{o x}=$ 0.21 again for the first $5 \mathrm{~s}$ of the simulation to establish a stabilized ambient flaming condition. After $t=5 \mathrm{~s}, X_{\mathrm{O}_{2}}^{o x}$ is decreased rapidly over a $3 \mathrm{~s}$ duration to a final specified value, which is then held constant for the remainder of the simulation.

Simulations are conducted using 40 processors on the University of Maryland Deepthought2 high-performance computing cluster. Typical simulations require roughly $2500 \mathrm{CPU}$ hours using Intel Ivy Bridge E5-2680v2 2.80 GHz processors. 
Table 1: Run-time characteristics for simulations with varying grid resolution.

\begin{tabular}{rrrr}
\hline$\Delta x(\mathrm{~mm})$ & \# Cells (-) & \# Cores (-) & CPU Time $(\mathrm{hr})$ \\
\hline 20.0 & 356,000 & 2 & 22 \\
10.0 & $1,056,000$ & 5 & 111 \\
5.0 & $6,400,000$ & 40 & 1,085 \\
2.5 & $51,200,000$ & 320 & 30,655 \\
\hline
\end{tabular}

\subsection{Resolution}

To evaluate grid sensitivity in the present configuration, a series of simulations have been conducted featuring uniform grid resolution corresponding to $\Delta x=20.0 \mathrm{~mm}, 10.0 \mathrm{~mm}, 5.0 \mathrm{~mm}$, and $2.5 \mathrm{~mm}$. These simulations are conducted with constant $X_{\mathrm{O}_{2}}^{o x}=0.21$ (no suppression) for a duration of $30 \mathrm{~s}$. Simulated diagnostics include vertical centerline ( $z$-direction), and lateral cross-flame profiles ( $x$-direction) of various locally computed quantities including gas-phase temperature, $z$-component of flow velocity, and the resolved and subgrid turbulent kinetic energies. Time-mean and root-mean-square (rms) statistics for each quantity are gathered over the final $25 \mathrm{~s}$ of each simulation, during a period when the flow is statistically stationary. Run-time characteristics for these simulations are summarized in Table 1, comparing the total number of cells, processors, and computational cost applicable to each resolution case.

Figure 3 presents comparisons of vertical centerline and cross-flame profiles of the time-mean and rms gas temperature, $T$, among each resolution case. As shown, adequate grid convergence with a mean relative deviation less than $5 \%$ is observed for $\Delta x \leq 5.0 \mathrm{~mm}$ in the mean temperature field. However, $T_{r m s}$ is under-predicted on the order of $50-100 \mathrm{~K}$ compared to the finest resolved case at $\Delta x=2.5 \mathrm{~mm}$. These results indicate that there remain unresolved turbulent fluctuations in the temperature field for $\Delta x \geq 2.5 \mathrm{~mm}$, though these fluctuations negligibly impact the mean field. The simulated peak mean value of $T \approx 1200 \mathrm{~K}$ closely resembles a value of $1191 \mathrm{~K}$ predicted by an empirical 


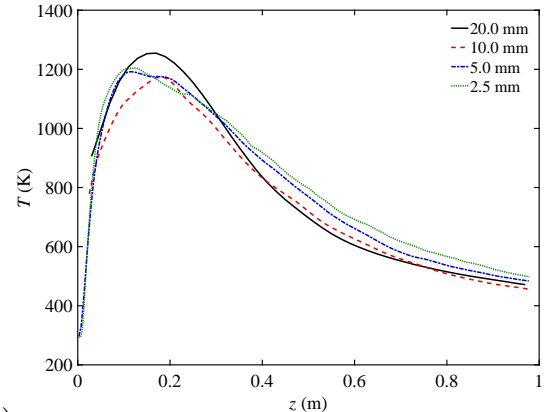

(a)

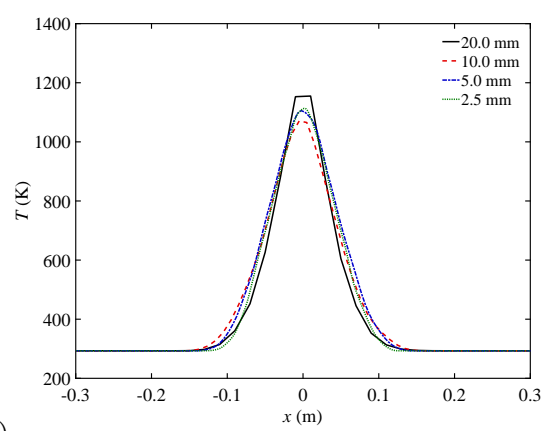

(b)
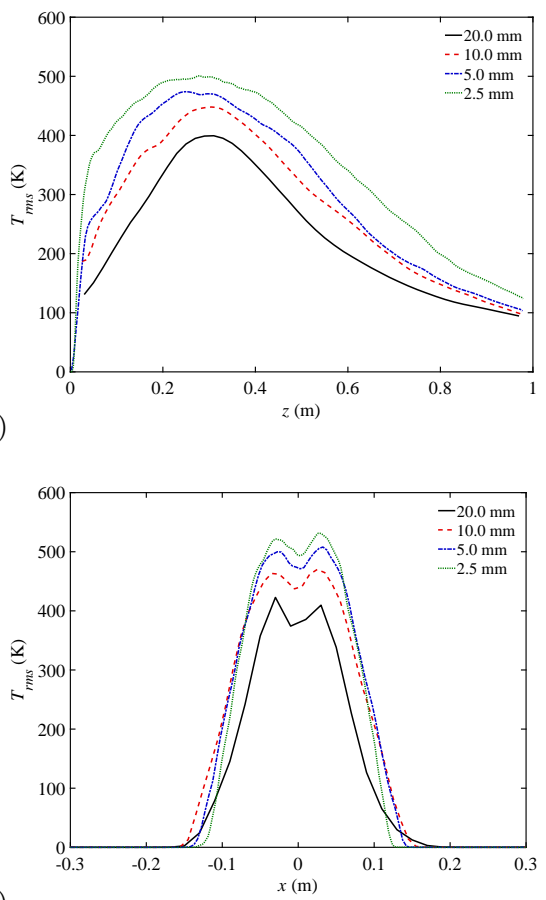

(d)

Figure 3: Simulated gas temperature $(T)$ at selected grid resolutions; (a) mean $z$-profile, (b) rms $z$-profile, (c) mean $x$-profile at $z=0.25 \mathrm{~m},(\mathrm{~d}) \mathrm{rms} x$-profile at $z=0.25 \mathrm{~m}$.

correlation developed for line-fire geometries from corresponding experimental measurements in a similar configuration [37].

Figure 4 presents similar resolution comparisons of the time-mean and rms $z$ velocity component magnitude, $w$. As shown, adequate grid convergence (mean relative deviation less than $5 \%$ ) is observed for $\Delta x \leq 5.0 \mathrm{~mm}$ in both the mean and rms values. As with the results for temperature, the simulated far-field value of $w \approx 2.8 \mathrm{~m} / \mathrm{s}$ agrees well with a value of $2.88 \mathrm{~m} / \mathrm{s}$ predicted by empirical correlation [37].

The mean turbulent kinetic energy resolved by the numerical grid, $k_{t}$, may be approximated as

$$
k_{t}=\frac{1}{2}\left(u_{r m s}^{2}+v_{r m s}^{2}+w_{r m s}^{2}\right),
$$

where $u_{r m s}, v_{r m s}$, and $w_{r m s}$, are respectively the root-mean-square statistics 

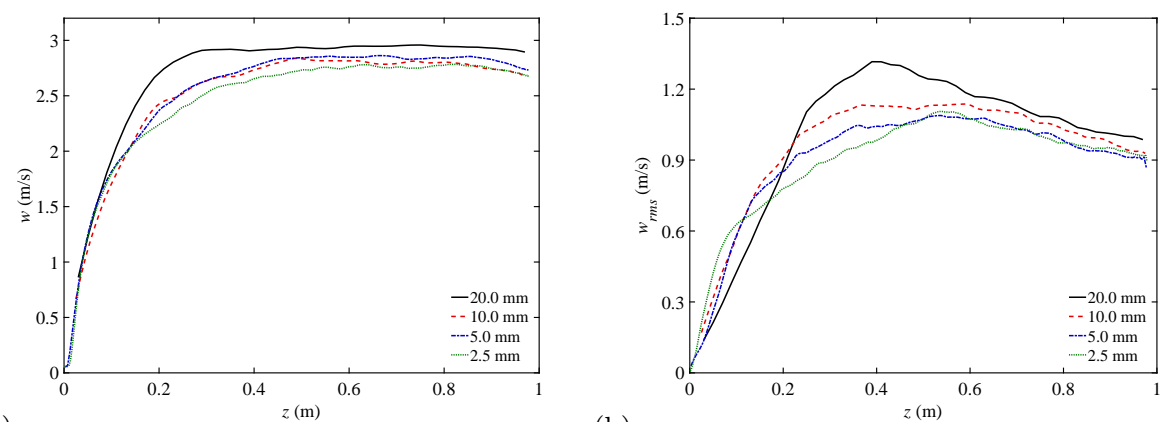

(a)

(b)
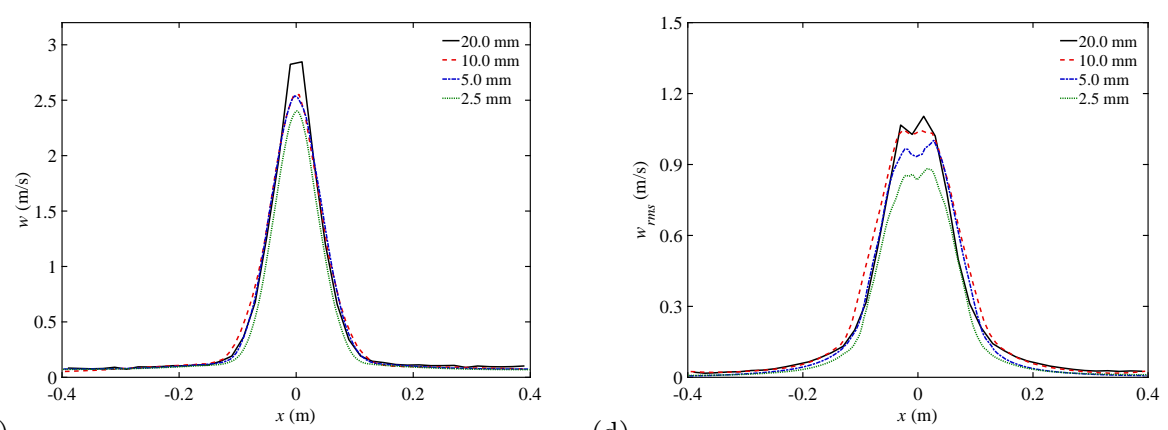

(c)

(d)

Figure 4: Simulated $z$-velocity magnitude $(w)$ at selected grid resolutions; (a) mean $z$-profile, (b) rms $z$-profile, (c) mean $x$-profile at $z=0.25 \mathrm{~m}$, (d) rms $x$-profile at $z=0.25 \mathrm{~m}$.

for the $x, y$, and $z$ components of the flow velocity. The unresolved subgrid turbulent kinetic energy, $k_{s g s}$, may then be retrieved from the turbulence model (see Eq. 5).

From these diagnostics, and following the conventions given in previous work [38], a characteristic resolution criterion, $M$, may be defined as

$$
M=\frac{k_{t}}{k_{t}+k_{s g s}}
$$

where this ratio indicates the fraction of the modeled turbulent kinetic energy that is resolved by the numerical grid, taking a value of unity when $k_{s g s}$ is negligible and decreasing toward zero as $k_{s g s}$ begins to dominate $k_{t}$. Note that $k_{s g s}$ is a modeled quantity and should not be expected to represent the true turbulent fluctuations in the unresolved flow. As a result, $M=1$ does not imply 


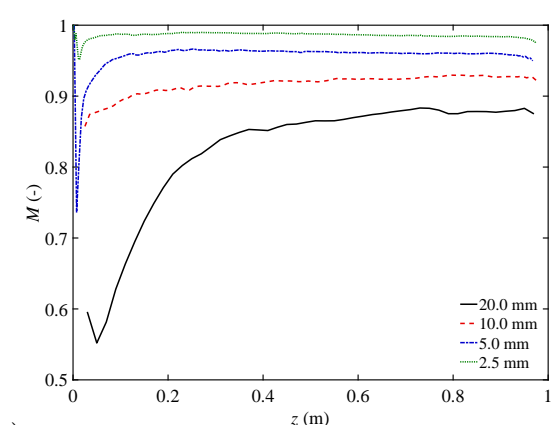

(a)

Figure 5: Simulated turbulence resolution criterion $(M)$ at selected grid resolutions; (a) mean $z$-profile, (b) mean $x$-profile at $z=0.25 \mathrm{~m}$.

that $100 \%$ of the turbulence in the flow is resolved by the grid. This criterion merely indicates the degree of influence that the turbulence model has on the flow field. Following established recommendations [36, 38], $M \geq 0.8$ satisfies reasonably well-resolved LES. Vertical centerline and cross-flame profiles of $M$ are presented in Fig. 5.

As shown in Fig. 5, for $\Delta x \leq 5.0 \mathrm{~mm}, M \geq 0.8$ for all $z$ along the flame centerline and for all $x$ along a representative cross-flame profile, though there is a small region very near to the fuel port for which $M<0.8$. This downward peak near $z=0.01 \mathrm{~m}$ is attributed to the laminar-turbulent transition that occurs at the flame base.

The limiting integral length scale of interest in the present configuration is the flame width $\left(W_{f}\right.$, approximated as the burner width, $\left.W_{b}=5 \mathrm{~cm}\right)$. For the baseline resolution of $\Delta x=5.0 \mathrm{~mm}, W_{b} / \Delta x=10$, indicating that this length scale is resolved over ten computational cells. In consideration of the previous results, this resolution may be considered marginal in the near-field region of the flame base (where $W_{f} \approx W_{b}$ ), but adequate in the intermediate and far-field regions within and above the bulk of the flame (where $W_{f}$ increases with elevation so that $W_{f}>10 \mathrm{~cm}$ and $W_{f} / \Delta x>20$ above mid-flame height, $z>0.25 \mathrm{~m})$. While it is questionable whether the $\Delta x=5.0 \mathrm{~mm}$ resolution accurately captures the transition from laminar to turbulent flow near the flame 
base, this resolution is deemed adequate and most cost-effective for the aims of the present study.

\subsection{Flame Extinction \& Reignition}

The fundamental limitation of any mixing-controlled combustion scheme (as presented in Eqs. 1-5), is that combustion is guaranteed to occur anywhere fuel and oxidizer are mixing, regardless of local conditions for temperature or fuel/oxidizer dilution. This represents the characteristic 'mixed-is-burned' treatment adopted by the EDC combustion framework and has the advantage that detailed heat transfer and chemical kinetics treatments are not necessary to model primary ignition at the fuel source (there is no need to specify a pilot in order for ignition to occur). As a result however, additional modeling provisions must be considered in order to account for flame extinction and prevent spurious reignition, each respectively addressed in the following sections.

\subsubsection{Extinction}

The baseline flame extinction algorithm available in FDS uses the concept of a critical flame temperature [11], which accounts for the thermal quenching mechanism, but neglects the aerodynamic and kinetic quenching mechanisms. For extinction via nitrogen dilution of the oxidizer in the present low-strain configuration, thermal quenching acts as the dominant mode of extinction; therefore a critical flame temperature based extinction model is sufficient in the present configuration.

The criterion for flame extinction in FDS incorporates an enthalpy balance where in order for combustion to occur, the potential heat release due to combustion must be sufficient to increase the mean temperature of the reactant mixture above a critical value, $T_{\text {ext }}$, defined as the critical flame temperature $[11,25]$. Combustion is allowed to proceed if the inequality given by

$$
\begin{aligned}
\hat{Y}_{\text {fuel }}\left(h_{\text {fuel }}(\tilde{T})+\Delta h_{\text {comb }}\right)+\hat{Y}_{\text {ox }} h_{\text {ox }}(\tilde{T})+\hat{Y}_{\text {dil }} h_{\text {dil }}(\tilde{T})> \\
\hat{Y}_{\text {fuel }} h_{\text {fuel }}\left(T_{\text {ext }}\right)+\hat{Y}_{\text {ox }} h_{\text {ox }}\left(T_{\text {ext }}\right)+\hat{Y}_{\text {dil }} h_{\text {dil }}\left(T_{\text {ext }}\right),
\end{aligned}
$$


is satisfied, where $\left[\hat{Y}_{f u e l}, \hat{Y}_{o x}, \hat{Y}_{d i l}\right]$ and $\left[h_{f u e l}, h_{o x}, h_{d i l}\right]$ are the mass fractions and mass-specific sensible enthalpies of the fuel, oxidizer (defined as air for the present study), and diluent (defined as any non-reactant species, including any inert agents or products of combustion) present in the reactant mixture, $\tilde{T}$ is the initial cell temperature, and $\Delta h_{c o m b}$ is the mass-specific enthalpy of combustion of the fuel. Note that Eq. 8 considers only the initial composition of the reactant mixture at the beginning of the combustion time step (after transport, but prior to any reaction). Extinction occurs if the local concentrations of fuel and oxidizer and/or the local cell temperature are too low to support combustion (thermal quenching). For further discussion of Eq. 8, the reader is referred to [11] and [32].

The reactant mixture referred in Eq. 8 represents the portion of the computational cell that is subject to potential combustion. The composition of the reactant mixture is defined relative to the composition of the host computational cell via the following,

$$
\begin{aligned}
\hat{Y}_{f u e l} & =\min \left(\tilde{Y}_{f u e l}, \tilde{Y}_{\text {ox }} / s\right), \\
\hat{Y}_{\text {ox }} & =s \hat{Y}_{\text {fuel }}, \\
\hat{Y}_{\text {dil }} & =\left(\hat{Y}_{\text {ox }} / \tilde{Y}_{\text {ox }}\right)\left(\tilde{Y}_{\text {fuel }}-\hat{Y}_{\text {fuel }}+\tilde{Y}_{\text {dil }}\right),
\end{aligned}
$$

where $\left[\tilde{Y}_{f u e l}, \tilde{Y}_{o x}, \tilde{Y}_{d i l}\right]$ are the local mass fractions of the fuel, oxidizer, and diluent in the computational cell.

By Eqs. 9-11, the reactant mixture comprises a stoichiometric combination of fuel and oxidizer, with additional diluent included such that the ratio of diluent to oxidizer in the reactant mixture matches that in the host computational cell $\left(\hat{Y}_{k}\right.$ is the mass of species $k$ in the stoichiometric reactant mixture divided by the total mass of the computational cell). These definitions (Eqs. 9-11) have the effect that excess fuel is treated as a diluent but excess oxidizer is not. As a result of this convention, combustion is likely to occur in a cell having a small amount of fuel in excess oxidizer, but is likely to be suppressed in a cell having a small amount of oxidizer in excess fuel. This treatment accommodates reason- 
able combustion and extinction performance in relatively coarse computational cells, as are frequently used in simulations of practical-scale fire configurations. For further discussion of Eqs. 9-11, the reader is referred to [11] and [32].

The critical flame temperature used in the present study, $T_{e x t}=1600 \mathrm{~K}$, is representative of typical hydrocarbon fuels [25]. If the criterion specified in Eq. 8 fails, combustion is suppressed and the reaction source terms for species generation/consumption and heat release are set to zero for that computational cell, at that time step $\left(\dot{Q}^{\prime \prime \prime}=\dot{m}_{k}^{\prime \prime \prime}=0\right.$ in Eqs. 1 and 2).

\subsubsection{Reignition}

By inspection of the combustion scheme presented in Eqs. 1-5, it is apparent that spurious reignition may be an issue anywhere fuel and oxidizer mix downstream of a localized extinction event. Including consideration for the previously defined extinction criterion (Eq. 8), reignition may occur as long as fuel and oxidizer are locally present in sufficient quantities to satisfy Eq. 8, which is possible even for low initial cell temperatures near ambient condition. An example of this fact is the initial ignition of primary combustion at the start of a simulation, which desirably does not require any specification for a pilot or other ignition heat source. Still, any non-physical, low-temperature reignition of previously extinguished fuel can significantly degrade the performance of the extinction model. These issues apply not only to the scheme presented in Eqs. 1-5, but more broadly to EDC combustion models in general, which are widely used in LES fire modeling applications. For applications in which flame extinction is the primary modeling interest, spurious reignition must be prevented.

Restricting available solutions, any reignition or extinction criterion that is implemented to prevent spurious reignition cannot be globally applied in the model without also suppressing primary ignition at the fuel source. In particular, preliminary simulations in the present configuration have found that simple pilots or transient heat sources are incapable of achieving self-sustained primary combustion of the fuel when a global reignition criterion is applied in 
the model. This issue may be circumvented by fully modeling primary ignition, though this would require an explicit ignition source coupled with detailed reaction kinetics and a highly-resolved treatment for heat transfer at the fuel source. Such an option is generally unavailable in fire applications which often include fuel sources that cannot be described with detailed reaction kinetics and complex configurations for which resolved heat transfer at the fuel source would require prohibitive computational cost. Despite these challenges, recent works have made notable progress in modeling and distinguishing ignition, extinction, and reignition processes in LES applications [14, 15].

Simulations in the present configuration highlight the noted issues surrounding spurious reignition, including conditions for which flame extinction leads directly to non-physical reignition if explicit treatment preventing such reignition is not provided in the model. As a preliminary modeling attempt, and following the convention of the critical flame temperature employed by the extinction criterion, a simple temperature based reignition criterion may be written as

$$
\tilde{T}>T_{i g n},
$$

where $\tilde{T}$ is the initial temperature of the computational cell (before the combustion time step) and $T_{i g n}$ is a critical temperature for reignition. As with the extinction criterion, if the inequality in Eq. 12 is unsatisfied, combustion is suppressed and the reaction source terms for species generation/consumption and heat release are set to zero for that cell, at that time step $\left(\dot{Q}^{\prime \prime \prime}=\dot{m}_{k}^{\prime \prime \prime}=0\right.$ in Eqs. 1 and 2). It should be noted that while Eq. 12 is relatively crude, its present use emphasizes the need for an explicit treatment of reignition and reflects the limited physical insight available to inform modeling choices for reignition phenomena. Equation 12 should be viewed as a preliminary modeling attempt that should be expected to improve with future research efforts.

As previously discussed, Eq. 12 cannot be generally applied in the solver without requiring an explicit treatment for primary ignition at the fuel source. To avoid this complication, implementations with selective application of Eq. 12 that still permit spontaneous primary ignition are explored in the following. 
Three simple modeling cases, respectively denoted M1, M2, and M3, and each utilizing Eqs. 8 and 12 to treat extinction and reignition in various capacities, are summarized as follows.

In each of the following cases, the baseline single-step global reaction scheme employed for primary combustion follows the EDC treatment and is defined as

$$
\text { Fuel }+s \text { Air } \rightarrow(1+s) \text { Products, }
$$

where reaction $\mathrm{R} 1$ is allowed to proceed only if the inequalities in both Eqs. 8 and 12 are satisfied, subject to the respective temperature criteria $T_{\text {ext }}$ and $T_{i g n}$.

The first case, M1, represents the standard EDC behavior for which $T_{\text {ext }}=$ $1600 \mathrm{~K}$ and $T_{i g n}=0 \mathrm{~K}$ everywhere in the computational domain. Here, Eq. 12 is universally satisfied such that M1 includes no provision to prevent spurious reignition. As previously stated, spurious reignition may occur if, downstream of a region where Eq. 8 is unsatisfied (and therefore reaction R1 is suppressed), the resulting unburned fuel propagates to a region where Eq. 8 is satisfied such that reaction $\mathrm{R} 1$ then spontaneously proceeds (potentially at non-physically low ignition temperatures).

The second case, M2, provides a novel development consisting of a simple extension of M1, but including a small prescribed ignition zone near the fuel source within which $T_{i g n}=0 \mathrm{~K}$. Elsewhere in the domain, $T_{i g n}=900 \mathrm{~K}$, a value approximately equal to an experimentally measured auto-ignition temperature for methane fuel [39]. Everywhere in the domain, $T_{\text {ext }}=1600 \mathrm{~K}$, consistent with M1. The ignition zone is defined within a region that extends $5 \mathrm{~mm}$ below, $5 \mathrm{~mm}$ around, and $2 \mathrm{~cm}$ above the surface of the fuel port. A diagram illustrating the bounds of the ignition zone is provided in Fig. 6 .

The spatially-variable application of $T_{i g n}$ employed by M2 effectively eliminates the issue of spurious reignition. Reignition is allowed to occur, but only if the local cell temperature is high enough to support such a process (via Eq. 12). M2 additionally provides the framework necessary to distinguish between piloted and non-piloted fuel sources, where for non-piloted applications, the ignition zone may be located remotely from the fuel source to model spontaneous 
ignition of a fuel leakage away from the leakage source.

The confined region of the ignition zone permits the convenience and simplicity of spontaneous primary ignition, but does so without subjecting the bulk of the computational domain to this convention. Despite these advantages, the specification of this zone is somewhat arbitrary and guidelines for its size and location do not yet exist. These are subjects of interest in the present work.

The third case, M3, follows conventions developed in recent affiliated works [14, 15], separating ignition and reignition using a tiered reaction mechanism. Including the primary reaction, $\mathrm{R} 1$, two additional reactions are introduced as

$$
\begin{gathered}
\text { Fuel }+s \text { Air } \rightarrow \text { Fuel }^{*}+s \text { Air } \\
\text { Fuel }^{*}+s \text { Air } \rightarrow(1+s) \text { Products, }
\end{gathered}
$$

where Fuel $^{*}$ is a secondary fuel species representing unburned fuel that has had the opportunity to react, but has been suppressed by Eq. 8. Here, reaction $\mathrm{R} 2$ acts as a mutually-exclusive alternate reaction pathway to reaction $\mathrm{R} 1$, transforming the primary Fuel into Fuel* if, and only if, R1 is suppressed. Reaction R3 then provides for the reignition of Fuel*, where the reignition criterion in Eq. 12 may be independently applied to R3 without affecting primary ignition in R1 (different $T_{i g n}$ may be separately applied to each reaction).

In M3, $T_{\text {ext }}$ and $T_{i g n}$ apply uniformly throughout the domain, with reactionspecific values defined as follows. For reaction $\mathrm{R} 1, T_{\text {ext }}=1600 \mathrm{~K}$ and $T_{i g n}=0 \mathrm{~K}$, equivalent to the treatment in M1. For reaction $\mathrm{R} 2, T_{\text {ext }}=0 \mathrm{~K}$ and $T_{i g n}=$ $0 \mathrm{~K}$, signifying that R2 is always unrestricted whenever R1 is suppressed. For

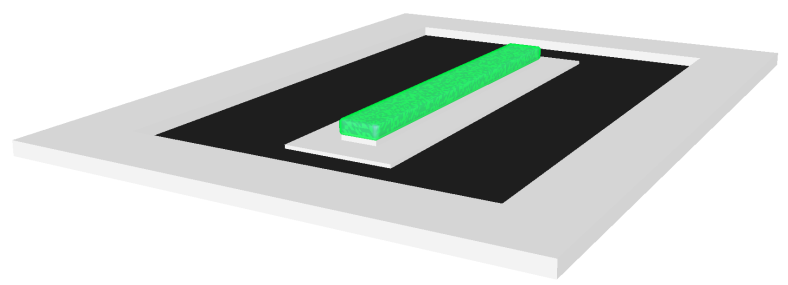

Figure 6: Diagram of the ignition zone applied in modeling case M2. 
Table 2: Critical temperature parameters for model cases M1, M2, and M3.

\begin{tabular}{cccc}
\hline Case & Reaction & $T_{\text {ext }}(\mathrm{K})$ & $T_{i g n}(\mathrm{~K})$ \\
\hline M1 & R1 & 1600 & 0 \\
\hline M2 & R1 & 1600 & 900 \\
\hline & R1 & 1600 & 0 \\
M3 & R2 & 0 & 0 \\
& R3 & 1600 & 900 \\
\hline
\end{tabular}

reaction $\mathrm{R} 3, T_{\text {ext }}=1600 \mathrm{~K}$ and $T_{i g n}=900 \mathrm{~K}$, matching the treatment in M2 (outside the ignition zone).

A summary of the critical temperature parameters, $T_{e x t}$ and $T_{i g n}$, as applied in cases M1, M2, and M3 is displayed in Table 2. For M1 and M2, the extinction and reignition criteria (Eqs. 8 and 12) are each applied only to reaction R1, whereas for M3, Eqs. 8 and 12 are each applied to R1, R2, and R3 independently. If either criterion fails, the applicable reaction source terms for species generation/consumption and heat release are set to zero for that computational cell, at that time step $\left(\dot{Q}^{\prime \prime \prime}=0 \dot{m}_{k}^{\prime \prime \prime}=0\right.$ in Eqs. 1 and 2).

The tiered reaction mechanism adopted in case M3 is advantageous in that it does not require an arbitrarily specified ignition zone. To its disadvantage however, M3 requires the additional complexity of reactions R2 and R3 and the computational expense of transporting an additional reacting species in Fuel*.

It is also important to recognize the implications of the stoichiometric requirement for air in the fuel transformation reaction, R2. This convention is necessary for the system of reactions R1-R3 to reduce back to the classical EDC treatment (R1) in cases of zero or total extinction; however, in situations where reaction $\mathrm{R} 1$ is extinguished and there is insufficient air to support reaction $\mathrm{R} 2$, fuel transformation will fail, permitting the persistence of the primary fuel in conditions of extinction, and providing the potential for spurious reignition to later occur (as visualized in the results that follow). While technically permissi- 
ble, this issue is limited to cases of extreme oxidizer dilution and in the majority of practical applications, M3 effectively eliminates spurious reignition.

\section{Results and Discussion}

\subsection{Model Validation}

A preliminary validation of each modeling case (M1, M2, and M3) against measured thermocouple temperatures $\left(T_{t c}\right)$ and local oxygen mole fractions $\left(X_{\mathrm{O}_{2}}\right)$ is presented in Fig. 7. Measured and simulated data are reported as lateral cross-flame profiles ( $x$-direction) at selected elevations corresponding to $z=12.5 \mathrm{~cm}\left(\sim L_{f} / 4\right)$ and $z=25.0 \mathrm{~cm}\left(\sim L_{f} / 2\right)$ above the fuel port, for a partially-diluted oxidizer condition of $X_{\mathrm{O}_{2}}^{o x}=0.18$. This validation condition serves to evaluate the influence that each extinction and reignition treatment may have on the flame structure for a partially suppressed condition where complete combustion is still expected.

As shown in Fig. 7, simulated $T_{t c}$ and $X_{O_{2}}$ for all three cases agree reasonably well with the measured values. In particular, peak $T_{t c}$ at both $z=12.5 \mathrm{~cm}$ and $z=25.0 \mathrm{~cm}$ show good agreement, though the simulated profiles are slightly wider than measured at both elevations. Agreement for $X_{O_{2}}$ at $z=12.5 \mathrm{~cm}$ is also good, though the simulated trends at $z=25.0 \mathrm{~cm}$ are slightly sharper than measured. These results validate that each case yields mean temperature and mixing fields that resemble those measured in the experiment, confirming that the simulated flame structure is not unreasonably skewed by the extinction and reignition treatments.

A preliminary verification test to evaluate the capacity for spurious reignition in each case is depicted in Fig. 8, which visualizes each simulated flame at $X_{\mathrm{O}_{2}}^{o x}=0$ (pure nitrogen co-flow). For this condition, total flame extinction is definitively expected with no possibility of reignition, corresponding to a simulated combustion efficiency of $\eta_{c o m b}=0$. Here, the simulated combustion efficiency is defined as

$$
\eta_{\text {comb }}=\frac{1}{\tau \dot{m}_{\text {fuel }} \Delta h_{\text {comb }}} \int_{\tau} \int_{V} \dot{Q}^{\prime \prime \prime} d V d t,
$$



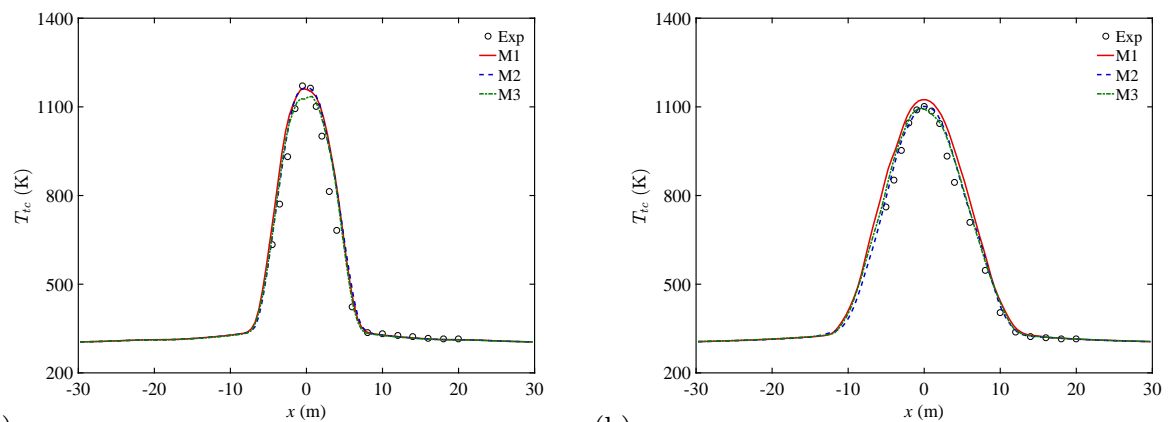

(a)

(b)
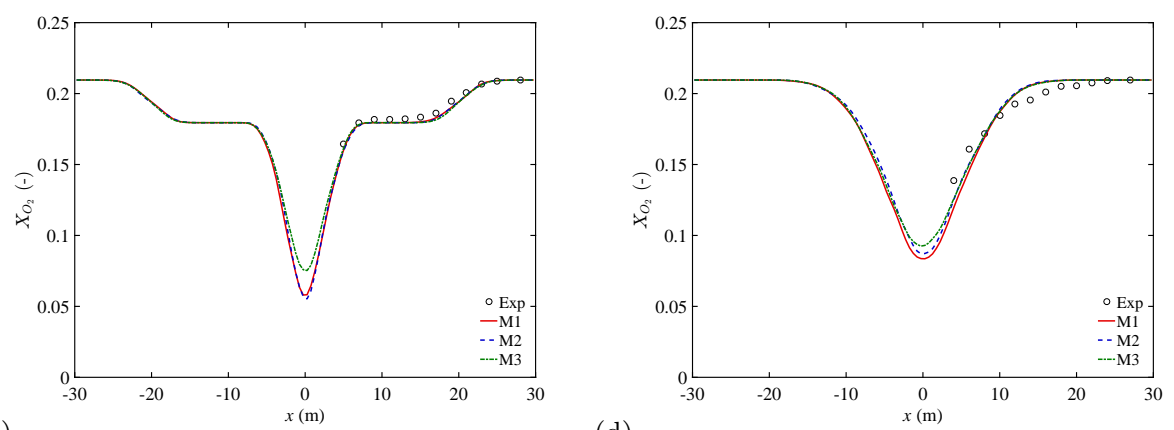

(c)

(d)

Figure 7: Simulated and measured mean $x$-profiles of local thermocouple temperature $\left(T_{t c}\right)$ and oxygen mole fraction $\left(X_{\mathrm{O}_{2}}\right)$ at selected elevations above the fuel port, and for $X_{\mathrm{O}_{2}}^{o x}=0.18$; (a) $T_{t c}$ at $z=12.5 \mathrm{~cm}$, (b) $T_{t c}$ at $z=25.0 \mathrm{~cm}$, (c) $X_{O_{2}}$ at $z=12.5 \mathrm{~cm}$, (d) $X_{O_{2}}$ at $z=25.0 \mathrm{~cm}$.

where $\dot{Q}^{\prime \prime \prime}$ is the local volumetric heat release rate in each computational cell, $\dot{m}_{f u e l}$ is the mass flow rate of primary fuel from the fuel port, $V$ is the volume of the computational domain, and $\tau$ is a time-averaging window.

For case M1 in Fig. 8(a), as expected, there is significant spurious reignition and $\eta_{c o m b}=0.69$. As shown, extinction is correctly predicted within the region of influence of the diluted oxidizer; however, as soon as the plume of unburned fuel mixes with sufficient air from the surrounding ambient (roughly $1 \mathrm{~m}$ above the fuel port) the inequality in Eq. (8) is satisfied and combustion proceeds spontaneously.

For case M2 in Fig. 8(b), there is no spurious reignition and $\eta_{\text {comb }}=0$. For 

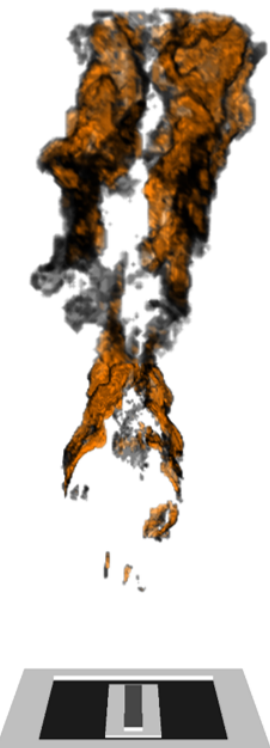

(a) (b)

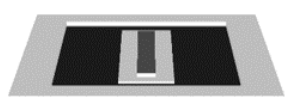

(c)

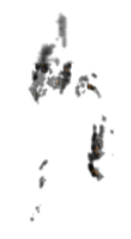

Figure 8: Spurious reignition in each modeling case for $X_{\mathrm{O}_{2}}^{o x}=0$; (a) M1 suffers significant spurious reignition $\left(\eta_{\text {comb }}=0.69\right)$, (b) M2 thoroughly eliminates spurious reignition $\left(\eta_{\text {comb }}=\right.$ $0)$, (c) M3 suffers slight, but nearly negligible spurious reignition $\left(\eta_{\text {comb }}=0.01\right)$. 


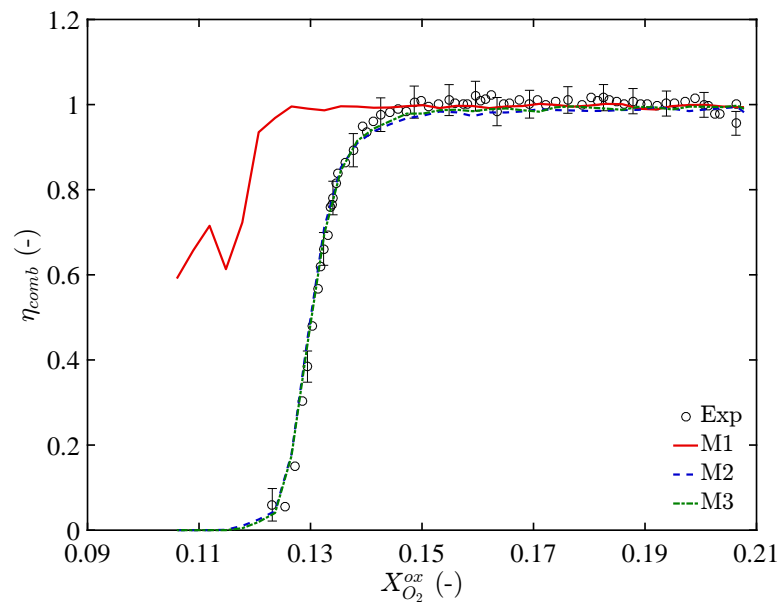

Figure 9: Simulated and measured combustion efficiency $\left(\eta_{c o m b}\right)$ plotted versus $X_{\mathrm{O}_{2}}^{o x}$; M1, which permits spurious reignition, fails to match the measured trend, while M2 and M3, which include provisions to prevent spurious reignition, match the measured data well.

$\eta_{c o m b} \approx 1$ for $X_{O_{2}}^{o x}>0.14$. Between $X_{O_{2}}^{o x}=0.14$ and $X_{O_{2}}^{o x}=0.125$, a sharp drop in $\eta_{c o m b}$ occurs, tapering briefly before global extinction $\left(\eta_{c o m b}=0\right)$, occurring at $X_{\mathrm{O}_{2}}^{o x} \approx 0.12$. Compared to the measured data, all simulated cases match the initial plateau in $\eta_{c o m b}$ for $X_{O_{2}}^{o x}>0.14$. For $X_{O_{2}}^{o x}<0.14$, M1 fails to follow the experimental trend and significantly over-predicts $\eta_{c o m b}$, primarily due to the occurrence of spurious reignition, as illustrated in Fig. 8(a). Notably, M1 does not predict deviation from $\eta_{c o m b} \approx 1$ until $X_{O_{2}}^{o x}<0.12$, where the experimental data (and M2 and M3) show global extinction. Also noteworthy, M1 fails to ever predict extinguishment.

As shown in Fig. 9, cases M2 and M3 follow the experimental $\eta_{\text {comb }}$ trend very well, falling within measurement uncertainty $( \pm 0.04)$, and matching the extinction limit at $L O I \approx 0.12$. Despite their different approaches at addressing spurious reignition (M2 via spatially defined ignition zone, and M3 via tiered reaction mechanism), the two cases also agree with one another remarkably well. In both cases, this agreement is achieved using relatively simple temperaturebased extinction and reignition treatments, with physically-relatable critical temperature values $\left(T_{e x t}=1600 \mathrm{~K}\right.$ and $\left.T_{i g n}=900 \mathrm{~K}\right)$. The results in Fig. 9 


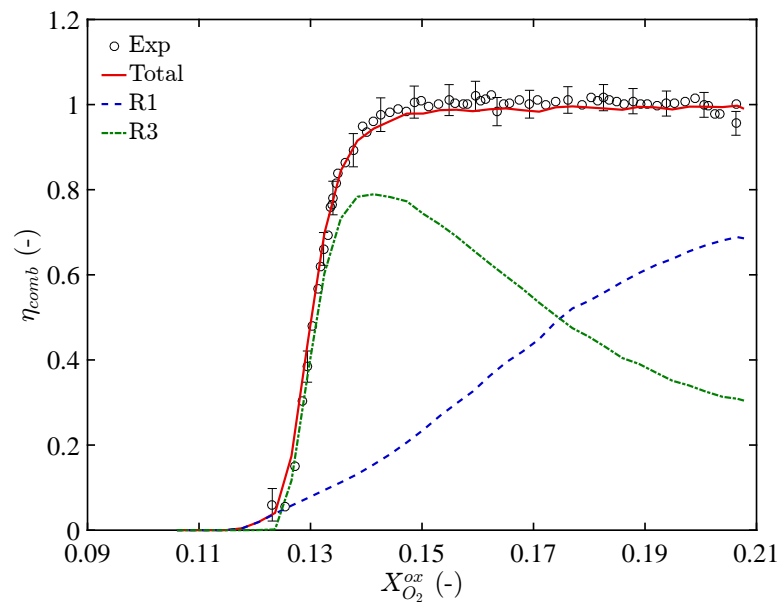

Figure 10: Simulated and measured combustion efficiency $\left(\eta_{\text {comb }}\right)$ among tiered reactions in modeling case M3; reaction R1 represents primary combustion, while reaction R3 represents reignition.

clearly demonstrate the significance of adequately preventing spurious reignition in simulations with localized flame extinction.

With its tiered reaction mechanism, case M3 permits additional detailed diagnostics to compare the relative contributions of heat release from either primary combustion (reaction R1) or reignition (reaction R3). These results are presented in Fig. 10. As shown, combustion is initially dominated by R1 at ambient $\mathrm{X}_{\mathrm{O}_{2}}^{o x}$, though there is considerable reignition heat release from $\mathrm{R} 3(\sim 30 \%)$, indicating that even at ambient conditions, M3 predicts localized extinction (though later reignition satisfies total $\eta_{c o m b} \approx 1$ ). As $X_{O_{2}}^{o x}$ is reduced, $\eta_{c o m b}, R 1$ steadily and monotonically decreases while $\eta_{c o m b}, R 3$ gradually increases. For $X_{O_{2}}^{o x}>0.14$, these trends offset, resulting in constant total simulated $\eta_{\text {comb }} \approx 1$. Near $X_{\mathrm{O}_{2}}^{o x} \approx 0.135, \eta_{c o m b}, R 3$ attains a peak value of roughly $80 \%$, dropping off abruptly with further declining $X_{\mathrm{O}_{2}}^{o x}$. Along with the continuous decline in $\eta_{c o m b, R 1}$, total $\eta_{c o m b}$ also decreases, tapering once $\eta_{c o m b, R 3}$ drops to zero, occurring just before global extinction.

In agreement with the results of recent affiliated work [15], these trends clearly demonstrate that reignition treatment can dominate simulated suppres- 
sion performance, where $\eta_{c o m b, R 3}$ is maximized and significantly greater than $\eta_{c o m b, R 1}$ throughout the region of rapidly declining $\eta_{c o m b}$ in advance of the extinction limit. The simulated result that $\eta_{c o m b}, R 3>0$ at ambient $X_{O_{2}}^{o x}$ additionally suggests that local extinction and reignition events may be prevalent in turbulent fires, even for unsuppressed conditions. While these insights are interesting, it must be noted that no experimental measurements presently exist which could corroborate any distribution of total heat release amongst primary combustion and reignition. The present results are then useful to evaluate model performance, but should not be construed to represent actual flame behaviors until supporting measurements are made available.

\subsection{Model Sensitivity}

The sensitivity of modeling cases M1, M2, and M3 to variations in input parameters is examined in Fig. 11, which primarily compares simulated $\eta_{c o m b}$ trends for each case among $T_{e x t}$ between $1500-1700 \mathrm{~K}$, and $T_{i g n}$ between $800-$ 1000 K. Specific to case M2, an additional sensitivity study considering the influence of the size of the prescribed ignition zone has also been performed.

Shown in Figs. 11(a), (c), and (e), respectively for cases M1, M2, and M3, a shift of $T_{e x t}$ by $100 \mathrm{~K}$ in either direction produces a corresponding shift in $L O I$ by roughly 0.015 . The same also results in a shift in the value of $X_{\mathrm{O}_{2}}^{o x}$ at the inflection point of $\eta_{c o m b}$ decline $\left(X_{O_{2}, \text { crit }}^{o x}\right)$ by roughly 0.015 , such that the entire $\eta_{c o m b}$ trend is synchronously shifted to higher or lower $X_{O_{2}}^{o x}$ with variation in $T_{\text {ext }}$. Similarly shown in Figs. 11(d) and (f), respectively for cases M2 and $\mathrm{M} 3$, a shift of $T_{i g n}$ by $100 \mathrm{~K}$ in either direction results in a negligible change in $L O I$, but a shift in $X_{O_{2}}^{o x}$,rit of roughly 0.01 (with M2 slightly more sensitive to variation in $T_{i g n}$ than M3). Shown in Fig. 11(b) (case M2), variations in ignition-zone elevation between $1.0-4.0 \mathrm{~cm}$ lead to a negligible change in $L O I$, with changes in $X_{O_{2}}^{o x}$, crit of less than 0.005. Simulated suppression performance in each case is then most sensitive to $T_{e x t}$, less sensitive to $T_{i g n}$, and for M2, even less sensitive to ignition zone size.

Note that while simulated suppression performance for case M2 is relatively 

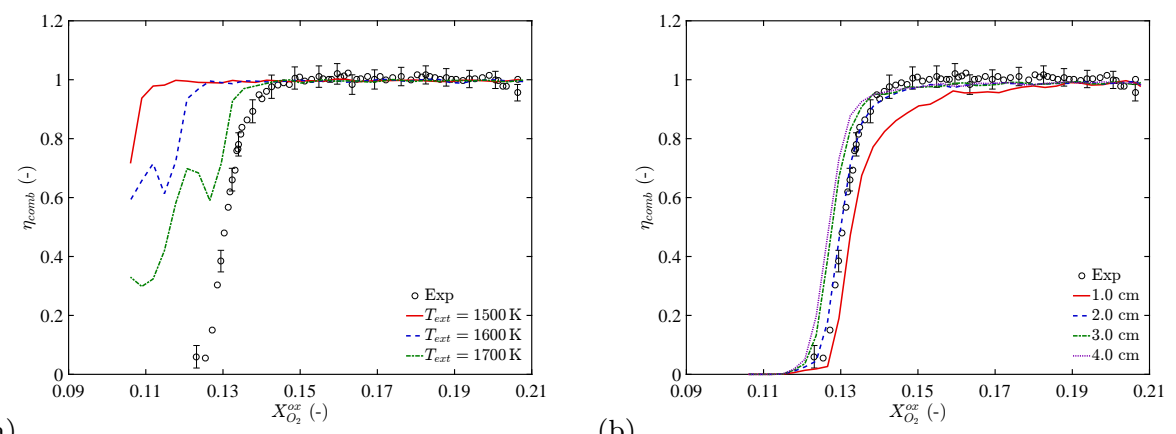

(a)

(b)

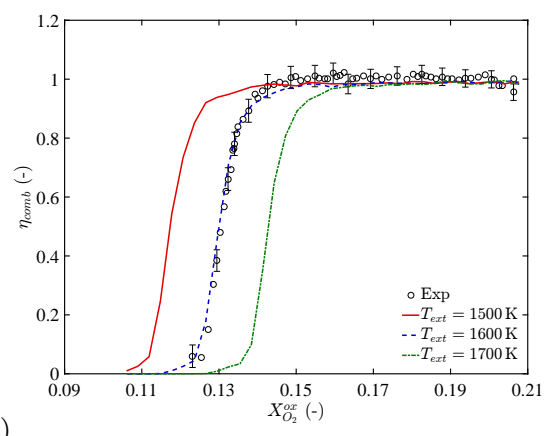

(c)

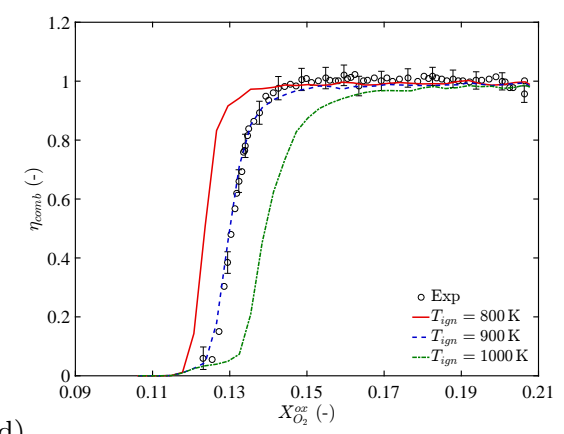

(d)

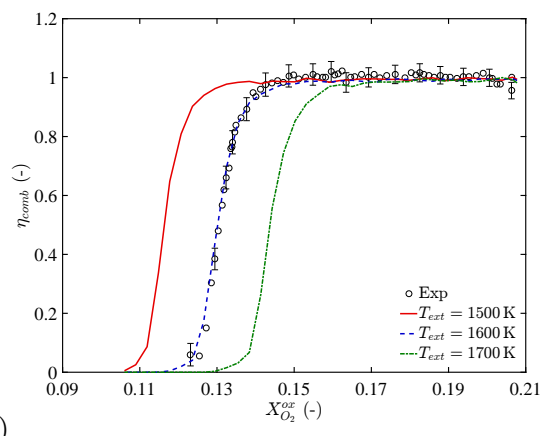

(f)

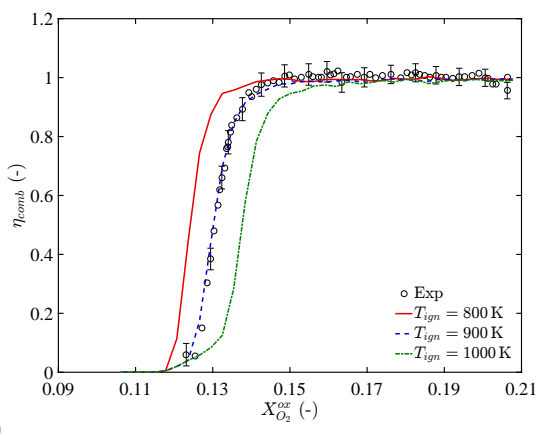

Figure 11: Sensitivity of simulated combustion efficiency $\left(\eta_{\text {comb }}\right)$ to varying model parameters; (a) M1 $T_{\text {ext }}$ sensitivity, (b) M2 ignition-zone size sensitivity, (c) M2 $T_{\text {ext }}$ sensitivity, (d) M2 $T_{i g n}$ sensitivity, (e) M3 $T_{\text {ext }}$ sensitivity, (f) M3 $T_{i g n}$ sensitivity. 
insensitive to increasing size of the ignition zone, as shown in Fig. 11(b), there does exist a minimum effective size for the ignition zone below which spontaneous primary ignition is not achieved. For the present configuration, this ineffectiveness limit corresponds to an ignition zone one computational-cell high, extending $5 \mathrm{~mm}$ above the fuel port. This minimum size is likely to depend on the configuration of the fuel source, the selected value for $T_{i g n}$, and the grid resolution (see Fig. 12 below), though no numerical or theoretical basis for its recommendation has yet been determined. A maximum size for the ignition zone however, may be discerned relative to the mean flame volume at ambient condition, where it would be desirable to limit the size of the ignition zone to be less than some small fraction of the mean flame volume.

The sensitivity of each modeling case to variations in grid resolution is explored in Fig. 12, including comparisons between the baseline resolution, $\Delta x=5.0 \mathrm{~mm}$, and coarsened resolutions, $\Delta x=10.0 \mathrm{~mm}$ and $\Delta x=20.0 \mathrm{~mm}$ (the refined resolution of $\Delta x=2.5 \mathrm{~mm}$ is deemed prohibitively expensive for the present analysis, with an estimated computational cost of greater than 100,000 processor-hours per simulation).

As depicted in Fig. 12(a), grid sensitivity in case M1 is relatively negligible, attributed to the dominance of non-physical spurious reignition over extinction within the domain. Comparatively, grid sensitivity in cases M2 and M3 is significant, respectively shown in Figs. 12(b) and (c). In both cases, grid coarsening leads to an over-prediction of suppression, attributed to the inherent grid sensitivity of the gas-phase cell temperature on which the reignition criterion is solely based and which tends to be under-predicted in coarse grids. Grid sensitivity is greatest in case M2, attributed to the bounds of the specified ignition zone, whose size does not scale with the underlying grid resolution so that for coarse grids, the relative size of the ignition zone becomes too small (contains too few cells) to support primary ignition.

Overall, the relatively high grid sensitivity of cases M2 and M3 is to be expected because the simplistic reignition treatment used in these cases relies on the grid-resolved gas-phase cell temperatures, which are also highly grid sensi- 


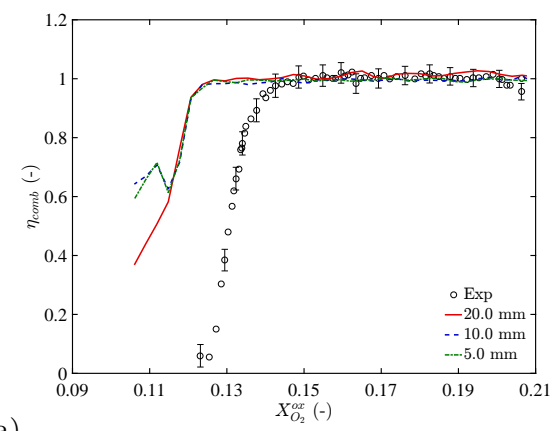

(a)

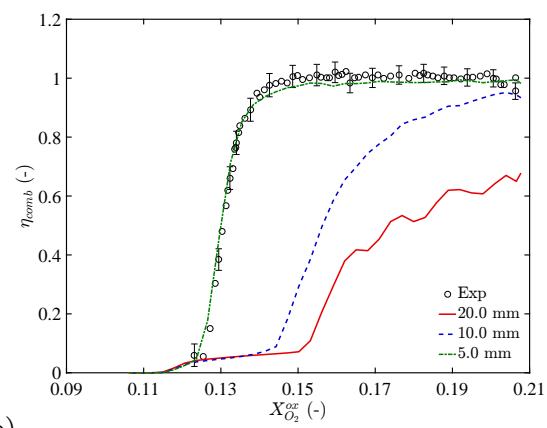

(b)

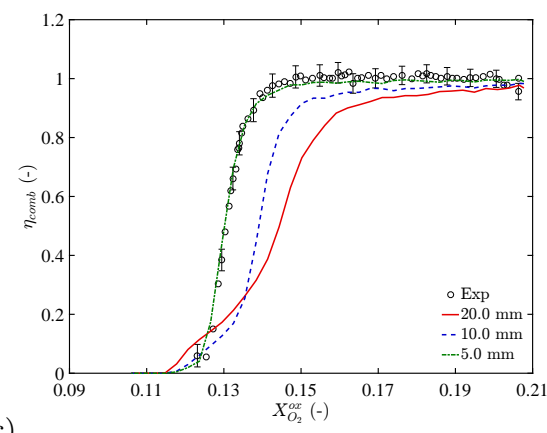

(c)

Figure 12: Sensitivity of simulated combustion efficiency $\left(\eta_{c o m b}\right)$ to varying grid resolution; (a) case M1, (b) case M2, (c) case M3. 
tive. As a result of this sensitivity, the proposed critical temperature parameters used in the extinction and reignition models, $T_{\text {ext }}$ and $T_{i g n}$, should not be viewed as generally applicable fuel properties. Instead, the performance of the extinction and reignition models, and therefore the appropriate choices for $T_{e x t}$ and $T_{i g n}$, are inexorably linked to the applicable configuration $\left(T_{\text {ext }}\right.$ and $T_{i g n}$ are dependent on the flow condition because strain related extinction effects are neglected) and to the selected grid resolution (clearly shown in Fig. 12).

While it must be recognized that such grid sensitivity is a significant detriment to the utility of the proposed reignition treatments, it must also be pointed out that superior alternative treatments applicable to LES simulations incorporating EDC combustion models have not yet been developed. The present models are therefore useful in light of lacking alternatives; however, improvement to the sensitivity of the proposed models or the development of superior alternatives should be a primary focus for future research.

\subsection{Model Performance}

Drawing from the present simulation results, a set of target objectives for extinction and reignition models applicable to fire safety engineering applications could include the following.

1. Primary fuel should be allowed to ignite spontaneously without the need to model complex ignition physics.

2. Combustion should be extinguished wherever extinction conditions are encountered, as determined by the chosen extinction model.

3. Following extinction events, fuel should not spuriously reignite once non extinction conditions are encountered elsewhere in the computational domain.

4. The model should ideally be free of arbitrary user input.

5. When desirable, the model should accommodate non-piloted fuel sources, such as fuel leakages.

Here, objective (1) is necessary for reasons described in Sec. 2.4.2, where the modeling of primary ignition is generally unavailable in fire applications, which 
often include fuels that cannot be described with detailed reaction kinetics and complex configurations for which resolved heat transfer at the fuel source would require prohibitive computational cost. The current state-of-the-art solution to objective (1) in the classical EDC combustion treatment is to completely neglect ignition, where fuel burns spontaneously upon mixing with oxidizer. This convention then necessitates objectives (2) and (3), respectively for the provision of flame extinction and the prevention of spurious reignition, where the significance of objective (3) is readily demonstrated in the simulation results presented in Sec. 3.1.

Objective (4) recognizes the desire for a model that requires no arbitrary or configuration-dependent user input, while objective (5) accommodates potential specialized applications whose interests may include the modeling of fuel transport through a domain with spontaneous ignition occurring at a specified location other than the location of fuel injection. Note that objectives (4) and (5) are likely to conflict with one another, as user input is sure to be necessary to distinguish between a fuel source where spontaneous ignition is desired and a fuel leakage, where spontaneous ignition may be undesirable.

Of the modeling cases considered in the present study, all incorporate EDC mixing-controlled combustion, by which objective (1) is satisfied. Each case additionally includes a simple extinction criterion based on the concept of a critical flame temperature (see Sec. 2.4.1), satisfying objective (2). As discussed in Sec. 2.4.2 (and demonstrated in Sec. 3.1), this combination of EDC combustion treatment with simplistic extinction modeling unfortunately leads to spurious reignition and thus the failure of objective (3) in case M1.

While case M2 passes objective (3), it does so with the requirement that a prescribed ignition region be explicitly specified within the computational domain. While a prescribed ignition zone may be desirable in some applications (as recognized in objective (5), which only M2 passes), its requirement will be undesirable in many more cases where the appropriate spatial bounds of the ignition zone are not easily discernible. In general, the size and shape of the ignition zone must relate to the physical configuration of the fuel source 
Table 3: Summary of objective performance for model cases M1, M2, and M3.

\begin{tabular}{cccc}
\hline Objective & M1 & M2 & M3 \\
\hline$(1)$ & $\checkmark$ & $\checkmark$ & $\checkmark$ \\
$(2)$ & $\checkmark$ & $\checkmark$ & $\checkmark$ \\
$(3)$ & $\times$ & $\checkmark$ & O \\
$(4)$ & $\checkmark$ & $\times$ & $\checkmark$ \\
$(5)$ & $\times$ & $\checkmark$ & $\times$ \\
\hline
\end{tabular}

and therefore no one-size-fits-all approach may be advised. Additional model sensitivities to the size of the ignition region and its performance dependency on grid resolution must also be considered (see Sec. 3.2). For these reasons, case M2 fails objective (4).

Case M3 addresses objective (3) using a tiered reaction mechanism (see Sec. 2.4.2), which avoids the constraint of arbitrary user input and therefore passes objective (4). Still, case M3 does require the added computational expense associated with the solution of an additional transport equation for the secondary fuel species. Additionally, though M3 adequately prevents spurious reignition in most situations, there are cases for which it fails to do so (see Fig. 8(c)). For this reason, M3 only qualifies objective (3).

The performance of each of the presently investigated extinction/reignition cases with respect to the stated modeling objectives is summarized in Table 3, where $\checkmark, \times$, and $O$ respectively indicate a passed, failed, or qualified objective.

From Table 3 , it is readily apparent that a simple and generally applicable approach to achieve accurate modeling of extinction and reignition within the limitations of the EDC combustion framework has not yet been developed. In the present configuration, for which objective (3) has been shown to be paramount to achieve reasonable simulated extinction performance, cases M2 and M3 (which pass or qualify this objective) are shown to yield accurate results when compared to measured global combustion efficiency data (see Fig. 9). 
While the reignition treatments adopted in these cases are simplistic and suffer from sensitivity issues (see Figs. 11 and 12), they produce significant improvements in model performance with manageable added complexity and computational cost.

The present results offer incentive to open new dialogues regarding the issue of spurious reignition in EDC combustion models, which until this investigation, has received limited or no attention in the broader research community, with all existing focus paid exclusively to extinction. The present results highlight that extinction modeling, while key to model performance, can be significantly affected and potentially dominated by reignition.

\section{Conclusions}

The present work provides a validation study to evaluate the capability of the Fire Dynamics Simulator (FDS), a large eddy simulation (LES)-based fire dynamics solver incorporating a mixing-controlled, eddy-dissipation concept (EDC) combustion framework to accurately describe flame response in a range of diluted oxidizer conditions, including a transition from complete combustion to total flame extinguishment. Three alternative cases for the implementation of critical flame temperature based extinction and reignition models are considered, including a baseline case without explicit reignition treatment (M1), a case including a novel spatially-variable reignition criterion (M2), and a case incorporating a tiered combustion mechanism with separate treatment of ignition, extinction, and reignition (M3). The extinction performance of these cases are compared against available experimental measurements, including local profiles of thermocouple temperature and oxygen mole fraction, as well as global combustion efficiency. Sensitivity studies for model input parameters and grid resolution are also provided.

In agreement with previous works, the present study reinforces the result that simulated extinction performance can be dominated by reignition. Comparisons of simulated results highlight the necessity for explicit treatment of 
reignition in mixing-controlled combustion models, where cases M2 and M3, which include provisions to prevent spurious reignition, yield extinction results that closely match the experimental data, while case M1, which does not restrict spurious reignition, fails to adequately predict flame extinction. While the reignition treatments adopted in cases M2 and M3 are relatively crude and suffer issues with grid sensitivity, they are shown to produce significant improvements in modeled extinction performance in the present configuration with manageable parametric sensitivity, added complexity, and computational expense. Future research endeavors are necessary to improve the present limitations of these types of models.

In comparing the extinction performance provided by each case, it is apparent that no simple and generally applicable approach is yet available to accurately model both extinction and reignition in LES models with mixingcontrolled EDC combustion. As a result, the requirement for configurationdependent user input is currently inevitable for certain applications using these types of models. In particular, additional in-flame experimental measurements are necessary to provide insight into the development of superior models and to better differentiate and determine which of the available models may best represent both extinction and reignition phenomena in general applications.

\section{Acknowledgments}

This project is supported by the U.S. National Science Foundation (NSFGOALI Award \#1236788). Conversations with P. Sunderland, E. Link, and T. Myers are gratefully acknowledged. The authors also acknowledge the computational resources at the University of Maryland and the NIST Fire Research Division, which were made available in conducting the research reported in this work.

\section{Nomenclature}




\begin{tabular}{|c|c|c|}
\hline mbol & & \\
\hline$C_{u}$ & model coefficient & $(-)$ \\
\hline$C_{v}$ & model coefficient & $(-)$ \\
\hline$h$ & mass-specific sensible enthalpy & $(\mathrm{J} / \mathrm{kg})$ \\
\hline$k$ & mass-specific kinetic energy & $\left(\mathrm{m}^{2} / \mathrm{s}^{2}\right)$ \\
\hline$L_{f}$ & flame height & $(\mathrm{m})$ \\
\hline$L O I$ & limiting oxygen index & $(\mathrm{mol} / \mathrm{mol})$ \\
\hline$\dot{m}$ & mass flow rate & $(\mathrm{kg} / \mathrm{s})$ \\
\hline$\dot{m}^{\prime \prime \prime}$ & mass reaction rate & $\left(\mathrm{kg} / \mathrm{m}^{3} / \mathrm{s}\right)$ \\
\hline$M$ & turbulence resolution criterion & $(-)$ \\
\hline $\operatorname{Pr}$ & Prandtl number & $(-)$ \\
\hline$\dot{Q}^{\prime \prime \prime}$ & volumetric heat release rate & $\left(\mathrm{W} / \mathrm{m}^{3}\right)$ \\
\hline$s$ & oxidizer to fuel mass ratio & $(\mathrm{kg} / \mathrm{kg})$ \\
\hline$S c$ & Schmidt number & $(-)$ \\
\hline$t$ & time & $(\mathrm{s})$ \\
\hline$T$ & temperature & $(\mathrm{K})$ \\
\hline$u$ & velocity, $x$-direction & $(\mathrm{m} / \mathrm{s})$ \\
\hline$v$ & velocity, $y$-direction & $(\mathrm{m} / \mathrm{s})$ \\
\hline$V$ & volume & $\left(\mathrm{m}^{3}\right)$ \\
\hline$w$ & velocity, $z$-direction & $(\mathrm{m} / \mathrm{s})$ \\
\hline$W$ & width & $(\mathrm{m})$ \\
\hline$X$ & mole fraction & $(\mathrm{mol} / \mathrm{mol})$ \\
\hline$Y$ & mass fraction & $(\mathrm{kg} / \mathrm{kg})$ \\
\hline$\Delta$ & LES filter width & $(\mathrm{m})$ \\
\hline$\Delta h_{\text {comb }}$ & mass-specific enthalpy of combustion & $(\mathrm{J} / \mathrm{kg})$ \\
\hline$\Delta h_{f}^{\circ}$ & mass-specific standard enthalpy of formation & $(\mathrm{J} / \mathrm{kg})$ \\
\hline
\end{tabular}




$\begin{array}{lll}{[\Delta x, \Delta y, \Delta z]} & \text { numerical grid resolution } & (-) \\ \eta_{\text {comb }} & \text { combustion efficiency } & (-) \\ \mu & \text { dynamic viscosity } & (\mathrm{kg} / \mathrm{m} / \mathrm{s}) \\ \rho & \text { mass density } & \left(\mathrm{kg} / \mathrm{m}^{3}\right) \\ \tau & \text { time-averaging window } & (\mathrm{s}) \\ \tau_{\text {mix }} & \text { turbulent mixing time scale } & (\mathrm{s}) \\ \chi_{\text {rad }} & \text { radiative loss fraction } & (-)\end{array}$

\section{Scripts}

$\begin{array}{ll}\infty & \text { ambient property } \\ b & \text { burner } \\ \text { crit } & \text { critical } \\ d i l & \text { diluent } \\ \text { ext } & \text { extinction } \\ f & \text { flame } \\ f u e l & \text { fuel } \\ \text { ign } & \text { ignition/reignition } \\ k & \text { indexing variable (species) } \\ \text { ox } & \text { oxidizer } \\ r m s & \text { root mean square } \\ \text { sgs } & \text { sub-grid scale } \\ t & \text { turbulent } \\ t c & \text { thermocouple }\end{array}$

714

715

716

717

718

References

[1] B. H. Chao, C. K. Law, J. S. T'ien, Structure and extinction of diffusion flames with flame radiation, Proceedings of the Combustion Institute 23 (1) (1991) 523-531.

[2] J. Du, R. L. Axelbaum, The effects of flame structure on extinction of $\mathrm{CH}_{4^{-}}$ 
$\mathrm{O}_{2}-\mathrm{N}_{2}$ diffusion flames, Proceedings of the Combustion Institute 26 (1) (1996) 1137-1142.

[3] S. H. Chan, J. Q. Yin, B. J. Shi, Structure and extinction of methane-air flamelet with radiation and detailed chemical kinetic mechanism, Combustion and Flame 112 (3) (1998) 445-456.

[4] J. L. Rhatigan, H. Bedir, J. S. T'ien, Gas-phase radiative effects on the burning and extinction of a solid fuel, Combustion and Flame 112 (1) (1998) 231-241.

[5] R. Chen, R. L. Axelbaum, Scalar dissipation rate at extinction and the effects of oxygen-enriched combustion, Combustion and Flame 142 (1) (2005) $62-71$.

[6] P. Narayanan, H. R. Baum, A. Trouvé, Effect of soot addition on extinction limits of luminous laminar counterflow diffusion flames, Proceedings of the Combustion Institute 33 (2) (2011) 2539-2546.

[7] V. Lecoustre, P. Narayanan, H. R. Baum, A. Trouvé, Local extinction of diffusion flames in fires, in: Fire Safety Science-Proceedings of the Tenth International Symposium, IAFSS, 2011, pp. 583-595.

[8] P. G. Arias, H. G. Im, P. Narayanan, A. Trouvé, A computational study of non-premixed flame extinction by water spray, Proceedings of the Combustion Institute 33 (2) (2011) 2591-2597.

[9] V. R. Lecoustre, P. G. Arias, S. P. Roy, Z. Luo, D. C. Haworth, H. G. Im, T. F. Lu, A. Trouvé, Direct numerical simulations of non-premixed ethylene-air flames: Local flame extinction criterion, Combustion and Flame 161 (11) (2014) 2933-2950.

[10] Z. Hu, Y. Utiskul, J. G. Quintiere, A. Trouvé, Towards large eddy simulations of flame extinction and carbon monoxide emission in compartment fires, Proceedings of the Combustion Institute 31 (2) (2007) 2537-2545. 
[11] J. Vaari, J. Floyd, R. McDermott, CFD simulations on extinction of coflow diffusion flames, in: Fire Safety Science-Proceedings of the Tenth International Symposium, IAFSS, 2011, pp. 781-793.

[12] A. Y. Snegirev, A. S. Tsoy, Treatment of local extinction in cfd fire modeling, Proceedings of the Combustion Institute 35 (3) (2015) 2519-2526.

[13] A. Y. Snegirev, Perfectly stirred reactor model to evaluate extinction of diffusion flame, Combustion and Flame 162 (10) (2015) 3622-3631.

[14] S. Vilfayeau, N. Ren, Y. Wang, A. Trouvé, Numerical simulation of under ventilated liquid-fueled compartment fires with flame extinction and thermally-driven fuel evaporation, Proceedings of the Combustion Institute 35 (3) (2015) 2563-2571.

[15] S. Vilfayeau, J. P. White, P. B. Sunderland, A. W. Marshall, A. Trouvé, Large eddy simulation of flame extinction in a turbulent line fire exposed to air-nitrogen co-flow, Fire Safety Journal 86 (2016) 16-31.

[16] J. C. Hewson, A. R. Kerstein, Local extinction and reignition in nonpremixed turbulent $\mathrm{CO} / \mathrm{H}_{2} / \mathrm{N}_{2}$ jet flames, Combustion Science and Technology 174 (5-6) (2002) 35-66.

[17] H. Pitsch, C. M. Cha, S. Fedotov, Flamelet modelling of non-premixed turbulent combustion with local extinction and re-ignition, Combustion Theory and Modelling 7 (2) (2003) 317-332.

[18] P. Sripakagorn, S. Mitarai, G. Kosály, H. Pitsch, Extinction and reignition in a diffusion flame: A direct numerical simulation study, Journal of Fluid Mechanics 518 (2004) 231-259.

[19] R. Venugopal, J. Abraham, A 2-D DNS investigation of extinction and reignition dynamics in nonpremixed flame--vortex interactions, Combustion and Flame 153 (3) (2008) 442-464. 
[20] R. Venugopal, J. Abraham, Numerical investigations of reignition in vortexperturbed n-heptane nonpremixed flames, AIAA Journal 46 (10) (2008) $2479-2497$.

[21] R. Venugopal, J. Abraham, Numerical studies of vortex-induced extinction/reignition relevant to the near-field of high-Reynolds number jets, Physics of Fluids 21 (5) (2009) 1-11.

[22] J. P. White, E. D. Link, A. C. Trouvé, P. B. Sunderland, A. W. Marshall, J. A. Sheffel, M. L. Corn, M. B. Colket, M. Chaos, H.-Z. Yu, Radiative emissions measurements from a buoyant, turbulent line flame under oxidizer-dilution quenching conditions, Fire Safety Journal 76 (2015) 74-84.

[23] J. P. White, E. D. Link, A. C. Trouvé, P. B. Sunderland, A. W. Marshall, A general calorimetry framework for measurement of combustion efficiency in a suppressed turbulent line fire, Fire Safety Journal, submitted for publication.

[24] Fire Dynamics Simulator, NIST, https://pages.nist.gov/fds-smv/ (2016).

[25] C. Beyler, Flammability limits of premixed and diffusion flames, in: P. DiNenno (Ed.), SFPE Handbook of Fire Protection Engineering, 4th Edition, National Fire Protection Association, 2008, pp. 194-210.

[26] B. F. Magnussen, B. H. Hjertager, On mathematical modeling of turbulent combustion with special emphasis on soot formation and combustion, Proceedings of the Combustion Institute 16 (1) (1977) 719-729.

[27] T. Poinsot, D. Veynante, Theoretical and Numerical Combustion, 2nd Edition, RT Edwards, Inc., 2005.

[28] R. J. McDermott, J. E. Floyd, Enforcing realizability in explicit multicomponent species transport, Fire Safety Journal 78 (2015) 180-187. 
[29] J. W. Deardorff, Stratocumulus-capped mixed layers derived from a threedimensional model, Boundary-Layer Meteorology 18 (4) (1980) 495-527.

[30] J. Bardina, J. H. Ferziger, W. C. Reynolds, Improved subgrid-scale models for large-eddy simulation, in: 13th Fluid and Plasma Dynamics Conference, AIAA, 1980, pp. 1-10.

[31] S. Hostikka, K. B. McGrattan, A. Hamins, Numerical modeling of pool fires using LES and finite volume method for radiation, in: Fire Safety ScienceProceedings of the Seventh International Symposium, IAFSS, 2003, pp. 383-394.

[32] K. McGrattan, S. Hostikka, R. McDermott, J. Floyd, C. Weinschenk, K. Overholt, Fire Dynamics Simulator Technical Reference Guide Volume 1: Mathematical Model, 6th Edition, NIST, 2016.

[33] K. McGrattan, S. Hostikka, R. McDermott, J. Floyd, C. Weinschenk, K. Overholt, Fire Dynamics Simulator Technical Reference Guide Volume 2: Verification, 6th Edition, NIST, 2016.

[34] K. McGrattan, S. Hostikka, R. McDermott, J. Floyd, C. Weinschenk, K. Overholt, Fire Dynamics Simulator Technical Reference Guide Volume 3: Validation, 6th Edition, NIST, 2016.

[35] R. J. McDermott, K. B. McGrattan, J. E. Floyd, A simple reaction time scale for under-resolved fire dynamics, in: Fire Safety Science-Proceedings of the Tenth International Symposium, IAFSS, 2011, pp. 809-820.

[36] S. B. Pope, Turbulent Flows, Cambridge University Press, 2000.

[37] L.-M. Yuana, G. Cox, An experimental study of some line fires, Fire Safety Journal 27 (2) (1996) 123-139.

[38] S. B. Pope, Ten questions concerning the large-eddy simulation of turbulent flows, New Journal of Physics 6 (35) (2004) 1-24. 
[39] C. Robinson, D. B. Smith, The auto-ignition temperature of methane, Journal of Hazardous Materials 8 (3) (1984) 199-203. 